\title{
Circle-Uniqueness of Pythagorean Orthogonality in Normed Linear Spaces
}

\author{
Senlin Wu, Xinjian Dong, and Dan Wang \\ Department of Applied Mathematics, Harbin University of Science and Technology, Harbin 150080, China \\ Correspondence should be addressed to Senlin Wu; wusenlin@outlook.com
}

Received 30 December 2013; Revised 5 February 2014; Accepted 7 February 2014; Published 11 March 2014

Academic Editor: Yongqiang Fu

Copyright (C) 2014 Senlin Wu et al. This is an open access article distributed under the Creative Commons Attribution License, which permits unrestricted use, distribution, and reproduction in any medium, provided the original work is properly cited.

We introduce the circle-uniqueness of Pythagorean orthogonality in normed linear spaces and show that Pythagorean orthogonality is circle-unique if and only if the underlying space is strictly convex. Further related results providing more detailed relations between circle-uniqueness of Pythagorean orthogonality and the shape of the unit sphere are also presented.

\section{Introduction}

We denote by $X=(X,\|\cdot\|)$ a real normed linear space whose dimension is at least 2 . The origin, unit ball, and unit sphere of $X$ are denoted by $o, B_{X}$, and $S_{X}$, respectively. When $X$ is twodimensional, it is called a Minkowski plane. Its unit sphere $S_{X}$ is then called the unit circle of $X$, and each homothetic copy of $S_{X}$ is a circle. For two distinct points (or vectors) $x$ and $y$ in $X$, we denote by $\langle x, y\rangle$ the line passing through $x$ and $y$, by $[x, y\rangle$ the ray starting from $x$ and passing through $y$, and by $[x, y]$ the (nondegenerate) segment connecting $x$ and $y$. Moreover, $X$ is said to be strictly convex if $S_{X}$ does not contain a nondegenerate segment.

Pythagorean orthogonality, which was introduced by James in [1], is one of the most natural extensions of orthogonality in inner product spaces to normed linear spaces (for other orthogonality types in normed linear spaces, we refer to [2-4] and the references therein). Let $x$ and $y$ be two vectors in a real normed linear space. If

$$
\|x-y\|^{2}=\|x\|^{2}+\|y\|^{2},
$$

then $x$ and $y$ are said to be Pythagorean orthogonal to each other (denoted by $x \perp_{P} y$ ). James showed that the following facts are equivalent:

(1) $x, y \in X, \alpha \in \mathbb{R}, x \perp_{P} y \Rightarrow x \perp_{P} \alpha y$;

(2) $X$ is an inner product space.
In other words, Pythagorean orthogonality is not homogeneous in general normed linear spaces. Among other things, James proved the line-existence of Pythagorean orthogonality: for each pair of vectors $x$ and $y$ in $X$, there exists a number $\alpha$ such that $x \perp_{P} \alpha x+y$. That is, James proved that in each line parallel to the line $\langle-x, x\rangle$ there exists a vector that is Pythagorean orthogonal to $x$.

However, James did not obtain any essential result on the uniqueness of this orthogonality type. Kapoor and Prasad [5] fixed this gap by proving that Pythagorean orthogonality is line-unique in each normed linear space $X$, where a binary relation $\perp$ on $X$ is said to be line-unique if and only if for each $x \neq o$ and $y \in X$ there exists a unique real number $\alpha$ such that $x \perp \alpha x+y$. It appears that the uniqueness of Pythagorean orthogonality has nothing to do with the shape of the unit ball. By introducing the circle-uniqueness (see Definition 1 next) of Pythagorean orthogonality, we show that this is not true. Our main result shows that Pythagorean orthogonality is circle-unique if and only if $X$ is strictly convex, which updates the knowledge about uniqueness of Pythagorean orthogonality.

For each $x \in X$, we denote by $P(x)$ the set of points that are Pythagorean orthogonal to $x$; that is,

$$
\begin{aligned}
P(x) & :=\left\{z \in X: z \perp_{P} x\right\} \\
& =\left\{z:\|z-x\|^{2}=\|z\|^{2}+\|x\|^{2}\right\} .
\end{aligned}
$$


For two linearly independent vectors $x$ and $y$ we denote by $L_{x, y}$ the two-dimensional subspace of $X$ spanned by $x$ and $y$ and by $H_{x, y}$ the closed halfplane of $L_{x, y}$ bounded by the line $\langle-x, x\rangle$ and containing $y$.

Definition 1. Pythagorean orthogonality on $X$ is said to be circle-unique if, for each pair of linearly independent vectors $x$ and $y$ and each nonnegative real number $\alpha$, there exists a unique vector $z$ in $\alpha S_{X} \cap H_{x, y} \cap P(x)$.

\section{Results and Proofs}

The following lemma concerning the intersection of two circles in a Minkowski plane is one of our main tools.

Lemma 2 (Theorem 2.4 in [6]). Let $C_{1}:=\gamma_{1} S_{X}+c_{1}$ and $C_{2}:=\gamma_{2} S_{X}+c_{2}$ be two circles in a Minkowski plane $X$, where $c_{1}$ and $c_{2}$ are two distinct points, and let $p$ and $q$ be the points of intersection of $\left\langle c_{1}, c_{2}\right\rangle$ and $C_{1}$. Then the set $C_{1} \cap C_{2}$ has one of the following forms:

(1) $C_{1} \cap C_{2}=\emptyset$;

(2) $C_{1} \cap C_{2}$ is the union of two closed, disjoint segments (one or both of them may degenerate to a singleton) lying on the opposite sides of $\left\langle c_{1}, c_{2}\right\rangle$;

(3) $C_{1} \cap C_{2}$ is the union of two segments (one or both of them may degenerate to a singleton) with common point $p$ or $q$.

One can easily verify the following proposition.

Proposition 3. Let $x$ and $y$ be two points in $X$. Then $x \perp_{P} y$ if and only if

$$
y \in\left(\sqrt{\|x\|^{2}+\|y\|^{2}} S_{X}+x\right) .
$$

First we show that Pythagorean orthogonality has the circle-existence property. More precisely, we show the following proposition.

Proposition 4. For each pair of linearly independent vectors $x$ and $y$ and each number $\alpha \geq 0$, the set

$$
P:=\alpha S_{X} \cap H_{x, y} \cap P(x)
$$

is a nonempty segment that may degenerate to a singleton.

Proof. We only consider the nontrivial case $\alpha>0$. Clearly,

$$
\begin{aligned}
P^{\prime} & :=L_{x, y} \cap\left(\alpha S_{X}\right) \cap P(x) \\
& =L_{x, y} \cap\left(\alpha S_{X}\right) \cap\left(\sqrt{\|x\|^{2}+\alpha^{2}} S_{X}+x\right) \\
& =\left(L_{x, y} \cap\left(\alpha S_{X}\right)\right) \cap\left(L_{x, y} \cap\left(\sqrt{\|x\|^{2}+\alpha^{2}} S_{X}+x\right)\right) .
\end{aligned}
$$

Since

$$
\sqrt{\|x\|^{2}+\alpha^{2}}-\alpha<\|x\|<\sqrt{\|x\|^{2}+\alpha^{2}}+\alpha,
$$

$P^{\prime}$ is not empty. It is also clear that $P^{\prime} \cap\langle-x, x\rangle=\emptyset$. Thus, by Lemma $2, P^{\prime}$ is the union of two closed, disjoint segments contained in $L_{x, y}$, one or both of which may degenerate to a singleton, lying in opposite halfplanes with respect to the line $\langle-x, x\rangle$. This completes the proof.

Next we state a simple result on common supporting lines of two circles.

Lemma 5. Let $X$ be a Minkowski plane, $x \neq 0$ a vector in $X$, and $\alpha, \beta>0$ two numbers such that

$$
0<\beta-\alpha<\|x\|
$$

Then there are two common supporting lines of $\alpha B_{X}$ and $\beta B_{X}+$ $x$ passing through the point $p=(\alpha /(\alpha-\beta)) x$.

Proof. By the hypothesis of the lemma, $p$ is exterior to $\alpha B_{X}$. Thus two supporting lines $l_{1}$ and $l_{2}$ of $\alpha B_{X}$ can be drawn through $p$. In the following we show that these two lines are two common supporting lines of $\alpha B_{X}$ and $\beta B_{X}+x$.

Clearly, there exists a point $u \in S_{X}$ such that $l_{1}$ supports $\alpha B_{X}$ at $\alpha u$. Put $\lambda_{0}=(\alpha-\beta) / \alpha$. Then

$$
\begin{aligned}
\lambda_{0} p+\left(1-\lambda_{0}\right) \alpha u & =\frac{\alpha-\beta}{\alpha} \cdot \frac{\alpha}{\alpha-\beta} x+\frac{\beta}{\alpha} \alpha u \\
& =\beta u+x \in \beta S_{X}+x
\end{aligned}
$$

which implies that $l_{1}$ intersects $\beta S_{X}+x$ in $\beta u+x$. Moreover,

$$
\begin{aligned}
\inf _{\lambda \in \mathbb{R}}\|x-(\lambda p+(1-\lambda) \alpha u)\| & \\
= & \inf _{\lambda \in \mathbb{R}} \| x-\lambda_{0} p-\left(1-\lambda_{0}\right) \alpha u+\lambda_{0} p \\
& \quad+\left(1-\lambda_{0}\right) \alpha u-(\lambda p+(1-\lambda) \alpha u) \| \\
= & \inf _{\lambda \in \mathbb{R}}\left\|\beta u-\left(\lambda_{0}-\lambda\right)(p-\alpha u)\right\| \\
= & \frac{\beta}{\alpha \lambda \in \mathbb{R}}\left\|\frac{\alpha}{\beta}\left(\lambda-\lambda_{0}\right) p+\frac{\alpha}{\beta}\left(\frac{\beta}{\alpha}-\left(\lambda-\lambda_{0}\right)\right) \alpha u\right\| \\
= & \beta\|u\| ;
\end{aligned}
$$

that is, the distance from $x$ to $l_{1}$ is $\beta\|u\|=\beta$. Thus $l_{1}$ is a common supporting line of $\alpha B_{X}$ and $\beta B_{X}+x$. In a similar way we can show that $l_{2}$ is also a common supporting line of these two discs.

Theorem 6. Let $x$ and $y$ be two linearly independent vectors, and let $\alpha$ be a positive number, $\beta=\sqrt{\alpha^{2}+\|x\|^{2}}$, and $p=$ $(1 /(\alpha-\beta)) x$. Then

$$
P:=\alpha S_{X} \cap H_{x, y} \cap P(x)
$$

is a nondegenerate segment if and only if there exist two unit vectors $u$ and $v$ in $H_{x, y}$ such that

(1) $[u, v]$ is a nondegenerate maximal segment contained in $S_{X} \cap H_{x, y}$; 
(2) $[v, u\rangle$ intersects $\langle-x, x\rangle$ at $p$;

(3) $\|u-v\| /\|u-p\|>\beta / \alpha-1$ or, equivalently, $\|\alpha u-\alpha v\|>$ $\|\beta u+x-\alpha u\|$.

Proof. It is clear that $p$ is exterior to $B_{X}$ and $\alpha p$ is exterior to $\alpha B_{X}$.

First suppose that $P$ is a segment $[m, n]$. Then

$$
[m, n] \subset \alpha S_{X} \cap\left(\beta S_{X}+x\right),
$$

which implies that $\langle m, n\rangle$ is one of the two common supporting lines of $\alpha B_{X}$ and $\beta B_{X}+x$. Lemma 5 shows that $\langle m, n\rangle$ intersects $\langle-x, x\rangle$ at $\alpha p$. Then there exist two unit vectors $u$ and $v$ such that

(1) $[u, v]$ is a maximal segment contained in $S_{X} \cap H_{x, y}$;

(2) $[(1 / \alpha) m,(1 / \alpha) n] \subseteq[u, v]$;

(3) $p \in[v, u\rangle \cap\langle-x, x\rangle$.

Thus there exists a number $\eta \in(0,1)$ such that $u=\eta p+(1-$ $\eta) v$. Since $[\beta u+x, \beta v+x]$ is the unique maximal segment contained in $\left(\beta S_{X}+x\right) \cap H_{x, y}$ and parallel to $[m, n]$, the lines $\langle m, n\rangle=\langle\alpha u, \alpha v\rangle$ and $\langle\beta u+x, \beta v+x\rangle$ coincide.

From

$$
\begin{gathered}
{[m, n] \subseteq[\alpha u, \alpha v] \subset \alpha S_{X},} \\
{[m, n] \subseteq[\beta u+x, \beta v+x] \subset \beta S_{X}+x,} \\
\beta u+x-\alpha p=\beta \eta p+\beta(1-\eta) v+x-\alpha p \\
=\beta \eta p+\beta(1-\eta) v-\beta p \\
=\beta(1-\eta)(v-p), \\
\alpha u-\alpha p=\alpha(1-\eta)(v-p)
\end{gathered}
$$

it follows that $\beta u+x \in[\alpha u, \alpha v] \backslash\{\alpha u, \alpha v\}$. Thus

$$
\begin{aligned}
\alpha\|u-v\| & =\|\alpha u-\alpha v\| \\
& >\|\beta u+x-\alpha u\|=(\beta-\alpha)\|u-p\| .
\end{aligned}
$$

Therefore, $u$ and $v$ are two unit vectors having the desired properties.

Conversely, suppose that $u$ and $v$ are two unit vectors having these properties. Clearly,

$$
\begin{gathered}
{[\alpha u, \alpha v] \subset \alpha S_{X} \cap H_{x, y},} \\
{[\beta u+x, \beta v+x] \subset\left(\beta S_{X}+x\right) \cap H_{x, y} .}
\end{gathered}
$$

Next we show that the lines $\langle\alpha u, \alpha v\rangle$ and $\langle\beta u+x, \beta v+x\rangle$ coincide. Since these two lines are parallel, we only need to show that they intersect. Clearly, there exists a number $\eta \in$ $(0,1)$ such that $u=\eta p+(1-\eta) v$ or, equivalently, $\alpha u=\eta \alpha p+$ $(1-\eta) \alpha v$. It follows that

$$
\begin{aligned}
\beta u+x & =\beta \eta p+\beta(1-\eta) v+x \\
& =\beta \eta p+(1-\eta)(\beta v+x)+\eta x \\
& =\eta(\beta p+x)+(1-\eta)(\beta v+x) \\
& =\eta \alpha p+(1-\eta)(\beta v+x) .
\end{aligned}
$$

Thus

$$
\alpha p \in\langle\alpha u, \alpha v\rangle \cap\langle\beta u+x, \beta v+x\rangle .
$$

In the rest of the proof we show that the intersection of the segments $[\alpha u, \alpha v]$ and $[\beta u+x, \beta v+x]$ is a nontrivial segment, which forces the set $P$ to be a nondegenerate segment. It suffices to show that $\beta u+x$ is a relatively interior point of the segment $[\alpha u, \alpha v]$.

On the one hand, we have

$$
\begin{aligned}
\beta u+x-\alpha p & =(1-\eta)(\beta v+x-\alpha p) \\
& =\beta(1-\eta)(v-p), \\
\alpha u-\alpha p & =\alpha(1-\eta)(v-p) .
\end{aligned}
$$

Thus, $\beta u+x$ lies in the set $[\alpha u, \alpha v\rangle \backslash\{\alpha u\}$. On the other hand, we have

$$
\begin{aligned}
\|\alpha u-\alpha v\| & =\alpha\|u-v\|>(\beta-\alpha)\|u-p\| \\
& =\|\beta u+x-\alpha u\| .
\end{aligned}
$$

It follows that $\beta u+x$ is from the relative interior of $[\alpha u, \alpha v]$.

Corollary 7. Let $x$ and $y$ be two linearly independent vectors and $\alpha$ be a positive number, $\beta=\sqrt{\alpha^{2}+\|x\|^{2}}$, and $p=(1 /(\alpha-$ $\beta)) x$. If $\alpha S_{X} \cap H_{x, y} \cap P(x)$ is a non-degenerate segment $[m, n]$, then there exist two unit vectors $u$ and $v$ such that $[u, v]$ is a maximal segment contained in $S_{X} \cap H_{x, y}$ and containing $[(1 / \alpha) m,(1 / \alpha) n],[v, u\rangle$ intersects $\langle-x, x\rangle$ at $p$, and

$$
\|u-v\|>\frac{\|x\|}{\alpha}+1-\frac{\beta}{\alpha} .
$$

Proof. Let $u$ and $v$ be defined as in the first part of the proof of Theorem 6. Then we only need to show (19). By the first part of the proof of Theorem 6 and the triangle inequality, we have

$$
\begin{aligned}
\|\alpha v-\alpha u\| & >\|\beta u+x-\alpha u\| \\
& =(\beta-\alpha) \| u+\frac{1}{\beta-\alpha} x \mid \\
& \geq(\beta-\alpha)\left|1-\frac{\|x\|}{\beta-\alpha}\right| \\
& =(\beta-\alpha)\left(\frac{\|x\|}{\beta-\alpha}-1\right) \\
& =\|x\|+\alpha-\sqrt{\alpha^{2}+\|x\|^{2}},
\end{aligned}
$$

from which (19) follows.

Corollary 8. Let $x$ and $y$ be two linearly independent vectors, $\alpha$ be a positive number, $\beta=\sqrt{\alpha^{2}+\|x\|^{2}}$, and $p=(1 /(\alpha-\beta)) x$. If $S_{X} \cap H_{x, y}$ contains a segment $\left[u^{\prime}, v^{\prime}\right]$ such that the ray $\left[v^{\prime}, u^{\prime}\right\rangle$ intersects the line $\langle-x, x\rangle$ at $p$ and that

$$
\left\|u^{\prime}-v^{\prime}\right\|>\frac{\beta}{\alpha}-1+\frac{\|x\|}{\alpha},
$$

then the set $\alpha S_{X} \cap H_{x, y} \cap P(x)$ is a nondegenerate segment. 
Proof. Let $u$ and $v$ be two unit vectors such that $\left[u^{\prime}, v^{\prime}\right] \subseteq$ $[u, v],[u, v]$ is a maximal segment contained in $S_{X} \cap H_{x, y}$, and $p \in[v, u\rangle \cap\langle-x, x\rangle$. By Theorem 6 , we only need to show $\|\alpha u-\alpha v\|>\|\beta u+x-\alpha u\|$. In fact,

$$
\begin{aligned}
\|\beta u+x-\alpha u\| & \leq \beta-\alpha+\|x\| \\
& =\alpha\left(\frac{\beta}{\alpha}-1+\frac{\|x\|}{\alpha}\right) \\
& <\alpha\left\|u^{\prime}-v^{\prime}\right\| \leq \alpha\|u-v\| \\
& =\|\alpha u-\alpha v\| .
\end{aligned}
$$

The proof is complete.

Now we have sufficient tools to prove the following theorem.

Theorem 9. Pythagorean orthogonality on $X$ is circle-unique if and only if $X$ is strictly convex.

Proof. If $X$ is strictly convex, then Corollary 7 shows that Pythagorean orthogonality is circle-unique.

Conversely, suppose that Pythagorean orthogonality is circle-unique. If $X$ is not strictly convex, then there exist two distinct unit vectors $u$ and $v$ in $X$ such that $[u, v] \subset S_{X}$. Let $\alpha>0$ be a number such that

$$
\|u-v\|>\frac{\sqrt{\alpha^{2}+1}}{\alpha}-1+\frac{1}{\alpha} .
$$

Put $\beta=\sqrt{\alpha^{2}+1}$. Since

$$
\frac{1}{\beta-\alpha}=\frac{1}{\sqrt{\alpha^{2}+1}-\alpha}=\sqrt{\alpha^{2}+1}+\alpha>1,
$$

the line $\langle u, v\rangle$ intersects $1 /(\beta-\alpha) S_{X}$ in a point $p$. By interchanging $u$ and $v$ if necessary, we may assume that $p \in$ $[v, u\rangle$. Put $x=(\alpha-\beta) p$. Then Corollary 8 implies that Pythagorean orthogonality on $X$ is not circle-unique, a contradiction.

In the end of this section we mention some result on the uniqueness of isosceles orthogonality, which was introduced by James in [1]: $x$ and $y$ are said to be isosceles orthogonal to each other if $\|x+y\|=\|x-y\|$. This orthogonality is not homogeneous in general normed linear spaces. The line-existence, line-uniqueness, circle-existence, and circle-uniqueness for isosceles orthogonality can be defined in a similar way. The uniqueness of isosceles orthogonality attracted much attention; see $[5,7,8]$. It has been shown that line-uniqueness and circle-uniqueness of isosceles orthogonality are equivalent to strict convexity of the underlying space. If $x$ and $y$ are two linearly independent vectors and $I(x)$ is the set of vectors isosceles orthogonal to $x$, then the property whether $\alpha S_{X} \cap$ $H_{x, y} \cap I(x)$ is a singleton is determined by the length of the segment (possibly degenerated to a point) contained in $S_{X} \cap L_{x, y}$ and parallel to $\langle-x, x\rangle$; see [8]. As we have shown, if $\alpha S_{X} \cap H_{x, y} \cap P(x)$ is not a singleton, then its structure is determined by a segment contained in $S_{X}$ which is not parallel to $\langle-x, x\rangle$. Moreover, for different values of $\alpha$, the segment determining the structure of $\alpha S_{X} \cap H_{x, y} \cap P(x)$ might be different.

\section{Conflict of Interests}

The authors declare that there is no conflict of interests regarding the publication of this paper.

\section{Acknowledgments}

The first named author is supported by the 973 program (Grant no. 2013CB834201), a foundation from the Ministry of Education of Heilongjiang Province (Grant no. 1251H013), the National Nature Science Foundation of China (Grant nos. 11371114 and 11171082), China Postdoctoral Science Foundation (Grant nos. 2012M520097 and 2013T60019), and the Scientific Research Foundation for the Returned Overseas Chinese Scholars, State Education Ministry.

\section{References}

[1] R. C. James, "Orthogonality in normed linear spaces," Duke Mathematical Journal, vol. 12, pp. 291-302, 1945.

[2] J. Alonso and C. Benítez, "Orthogonality in normed linear spaces: a survey. II. Relations between main orthogonalities," Extracta Mathematicae, vol. 4, no. 3, pp. 121-131, 1989.

[3] J. Alonso, H. Martini, and S. Wu, "On Birkhoff orthogonality and isosceles orthogonality in normed linear spaces," Aequationes Mathematicae, vol. 83, no. 1-2, pp. 153-189, 2012.

[4] J. Alonso, "Uniqueness properties of isosceles orthogonality in normed linear spaces," Annales des sciences mathématiques du Québec, vol. 18, no. 1, pp. 25-38, 1994.

[5] O. P. Kapoor and J. Prasad, "Orthogonality and characterizations of inner product spaces," Bulletin of the Australian Mathematical Society, vol. 19, no. 3, pp. 403-416, 1978.

[6] J. Banasiak, "Some contribution to the geometry of normed linear spaces," Mathematische Nachrichten, vol. 139, pp. 175-184, 1988.

[7] J. Alonso and C. Benítez, "Orthogonality in normed linear spaces: a survey. I. Main properties," Extracta Mathematicae, vol. 3, no. 1, pp. 1-15, 1988.

[8] D. Ji, J. Li, and S. Wu, "On the uniqueness of isosceles orthogonality in normed linear spaces," Results in Mathematics, vol. 59, no. 1-2, pp. 157-162, 2011. 


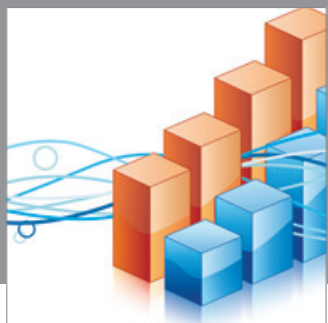

Advances in

Operations Research

mansans

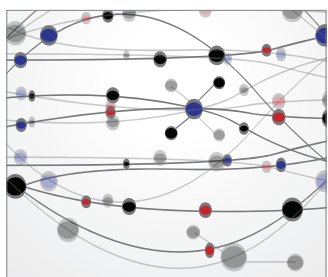

The Scientific World Journal
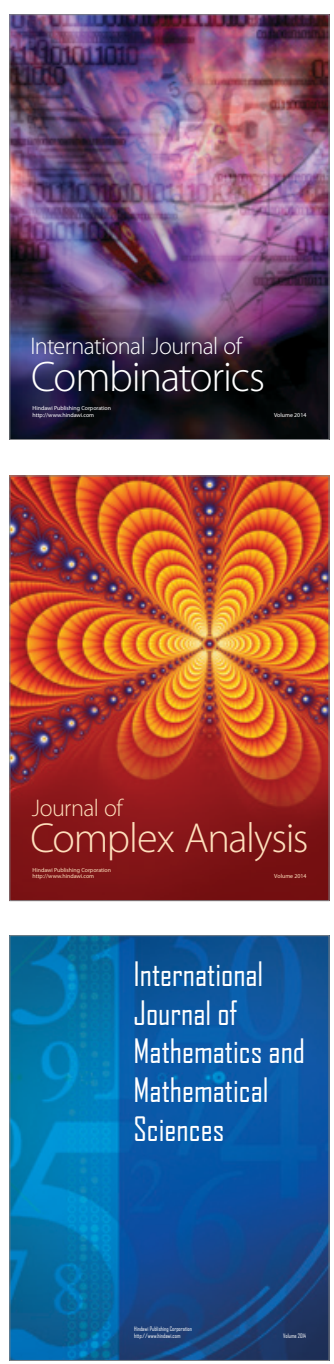
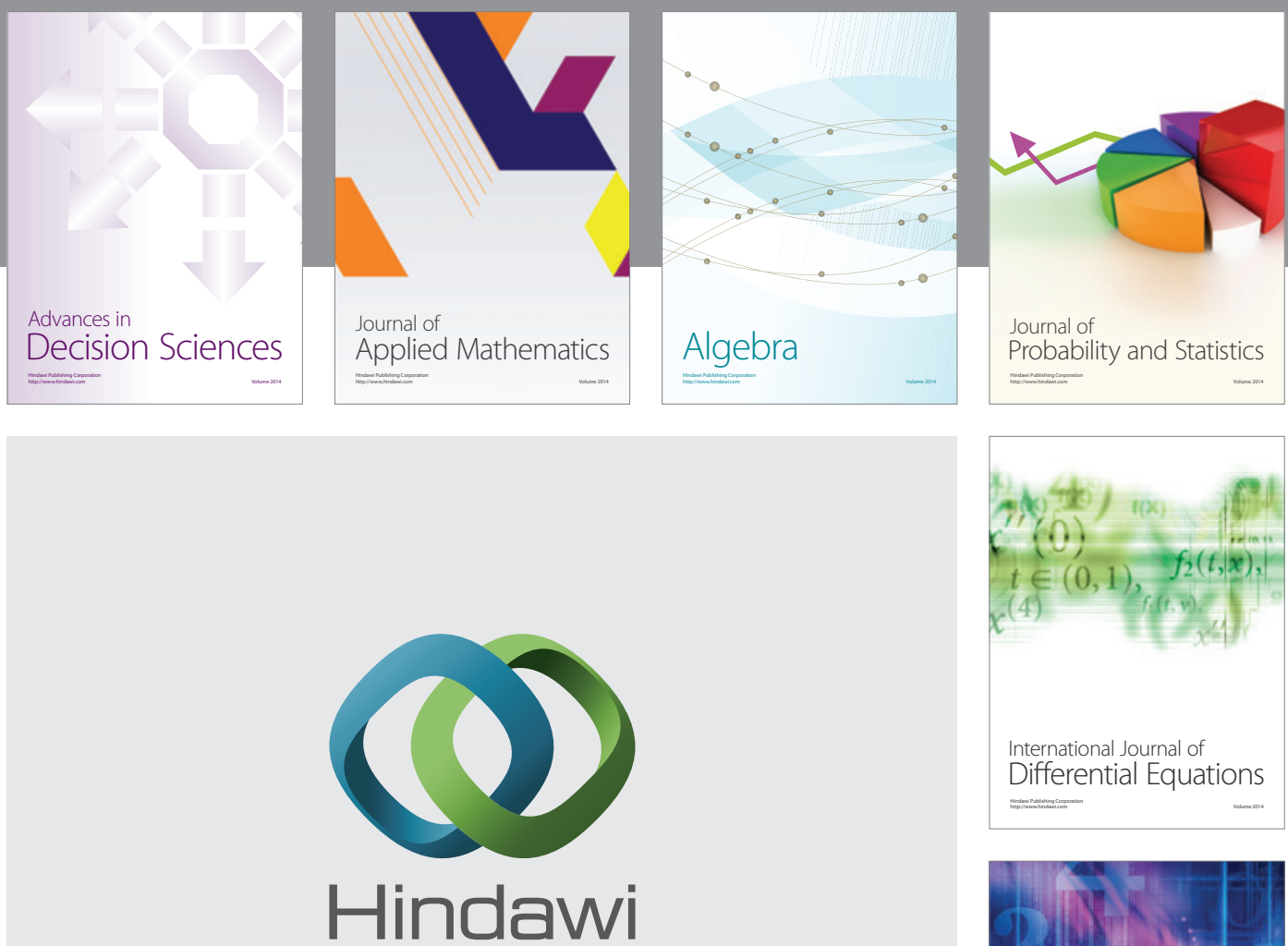

Submit your manuscripts at http://www.hindawi.com
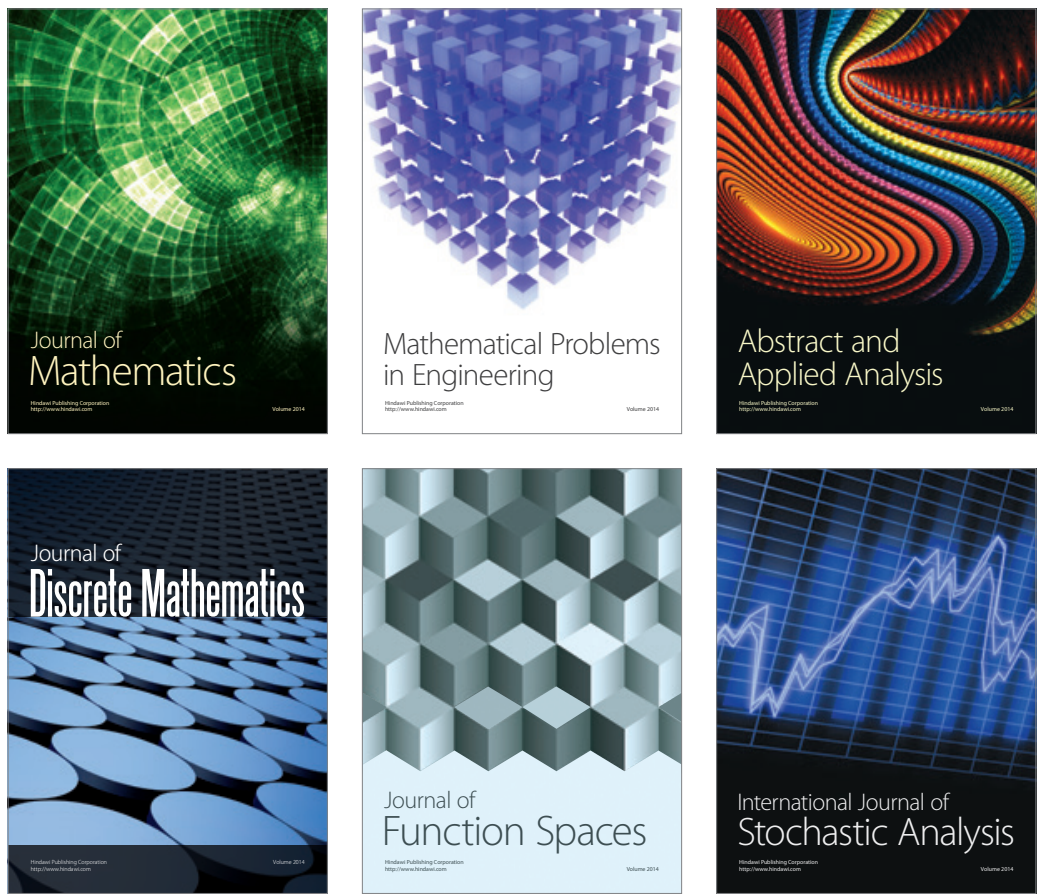

Journal of

Function Spaces

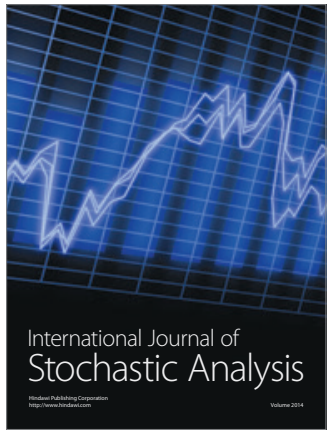

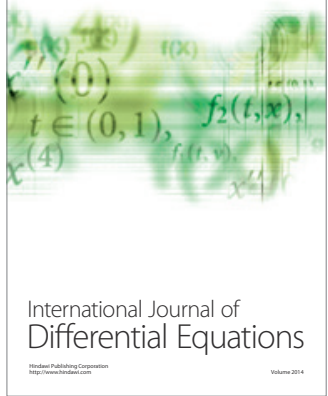
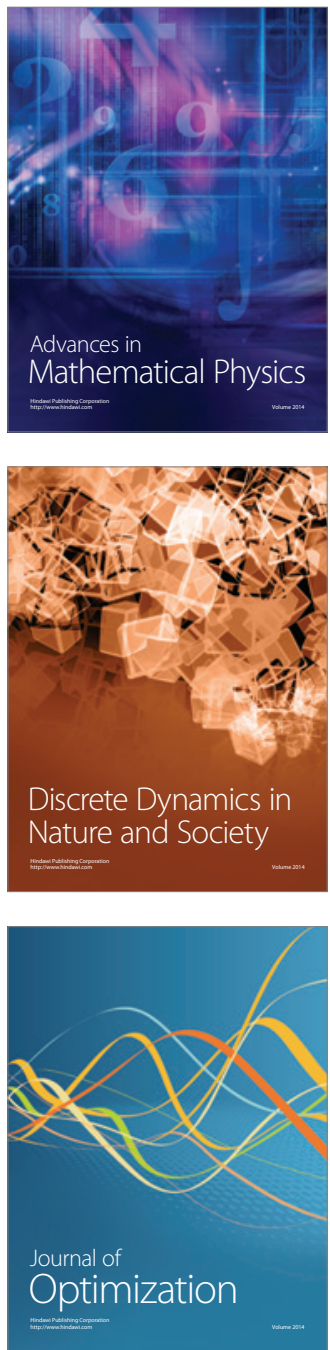